\title{
Radioimmunotherapy in Non-Hodgkin Lymphoma: Opinions of Nuclear Medicine Physicians and Radiation Oncologists
}

\author{
Niklaus G. Schaefer ${ }^{1}$, Peng Huang ${ }^{2}$, Julia W. Buchanan ${ }^{1}$, and Richard L. Wahl ${ }^{1}$ \\ ${ }^{1}$ Division of Nuclear Medicine, Russell H. Morgan Department of Radiology and Radiological Sciences, Johns Hopkins University \\ School of Medicine, Baltimore, Maryland; and ${ }^{2}$ Division of Oncology Biostatistics, Johns Hopkins University, Baltimore, Maryland
}

Despite approval by the Food and Drug Administration and consistent reports of the efficacy and safety of ${ }^{90} \mathrm{Y}$-ibritumomab tiuxetan and ${ }^{131} \mathrm{I}$-tositumomab, these therapies are infrequently used. This study investigates the opinions and patterns of the use of radioimmunotherapy by nuclear physicians, affiliated researchers, nuclear medicine technologists, and radiation oncologists and aims to identify possible barriers to the use of this promising therapy. Methods: An e-mail-based survey with 13 broad questions related to radioimmunotherapy was sent electronically to 13,221 Society of Nuclear Medicine members and radiation oncologists throughout the United States. Results: Six hundred thirteen individuals (4.6\%) responded to the electronic survey. Two hundred fifty-one responders $(40.9 \%)$ had treated patients with non-Hodgkin lymphoma (NHL) with radioimmunotherapy in the last 24 mo. Of the responders, $29.5 \%$ used only ${ }^{90}$ Y-ibritumomab tiuxetan, $7.6 \%$ used only ${ }^{131} \mathrm{I}$-tositumomab, and $24.9 \%$ used both radiopharmaceuticals; $37.9 \%$ did not treat $\mathrm{NHL}$ with radioimmunotherapy. Most responders said their patients came from university hospitals $(33.9 \%)$ or private offices $(25.6 \%)$, and they mainly treated in a second-line (42.9\%), third-line (35.6\%), or consolidation $(23.5 \%)$ setting. Major concerns were that referring oncologists and hematologists wanted to treat by themselves with nonradioactive compounds (mean $\pm \mathrm{SD}, 3.418 \pm 1.49$ ) and that ${ }^{90} \mathrm{Y}$-ibritumomab tiuxetan and ${ }^{131}$ I-tositumomab were expensive (mean $\pm \mathrm{SD}, 3.413 \pm 1.35$ ). Of the responders and involved physicians, $40.4 \%$ and $35.2 \%$, respectively, did not know if their institution accepted Medicare patients for radioimmunotherapy. Almost 30\% (29.6\%) of the responders thought radioimmunotherapy would probably grow and $38.0 \%$ thought it would grow in importance in the future. Responders who did not administer radioimmunotherapy for $\mathrm{NHL}$ thought it took too much time to administer radioimmunotherapy $(P<$ $0.01)$ and had concerns about the dosimetry procedure $(P<$ $0.01)$ and radiation safety $(P<0.01)$. Individuals who perceived a negative future for radioimmunotherapy had significantly more concerns about the time-consuming administration process $(P<0.05)$ and the high cost of radioimmunotherapy $(P<0.05)$. Responders from academic centers had significantly fewer concerns about payment $(P<0.01)$, dosimetry $(P<0.01)$, and radiation safety $(P<0.01)$. Conclusion: Radioimmunotherapy was generally viewed positively by the surveyed population. However,

\footnotetext{
Received Nov. 22, 2010; revision accepted Jan. 31, 2011.

For correspondence or reprints contact: Richard L. Wahl, Division of Nuclear Medicine/PET Center, Russell H. Morgan Department of Radiology and Radiological Sciences, Johns Hopkins University School of Medicine, Rm. 3223 JHOC, 601 N. Caroline St., Baltimore, MD 21287-0817.

E-mail: rwahl@jhmi.edu

COPYRIGHT @ 2011 by the Society of Nuclear Medicine, Inc.
}

limited referrals due to alternative nonradioactive therapies and logistic, educational, and economic concerns played an important role for subgroups in the perception of radioimmunotherapy for NHL.

Key Words: lymphoma; radioimmunotherapy; survey; pharmacoeconomics

J Nucl Med 2011; 52:830-838

DOI: 10.2967/jnumed.110.085589

\section{$\mathbf{T}$}

reatment with anti-CD20 monoclonal antibodies is the most significant recent contribution to the treatment of CD20-positive indolent non-Hodgkin lymphoma (NHL) (1). Several clinical studies have demonstrated the high efficacy of radioimmunotherapy of follicular low-grade NHL (2-7). Radioimmunotherapy regimens with ${ }^{90} \mathrm{Y}-$ ibritumomab tiuxetan (Zevalin; Spectrum Pharmaceuticals) and ${ }^{131}$ I-tositumomab (Bexxar; GlaxoSmithKline) are approved for treatment of patients with follicular and transformed NHL who failed or relapsed after prior therapies, including rituximab and standard chemotherapy. The recent publication of an 8-y follow-up of a multicenter trial using 6 cycles of standard chemotherapy followed by ${ }^{131}$ I-tositumomab shows a 5-y progression-free survival (PFS) and overall survival of $56 \%$ and $83 \%$, respectively. After more than $8 \mathrm{y}$, the median response duration has not been reached (8). A recent randomized multicenter phase III trial (Firstline Indolent Trial [FIT]) investigated ${ }^{90}$ Y-ibritumomab tiuxetan as a first-line consolidation therapy, given as a single therapeutic dose to patients with advanced (stage III or IV) follicular lymphoma who achieved at least a partial remission or complete remission after receiving various induction chemotherapy regimens. The results from 414 patients show a significantly prolonged median PFS of 13.3$36.5 \mathrm{mo}$. These promising results led to regulatory approval for ${ }^{90}$ Y-ibritumomab tiuxetan as a first-line consolidation therapy in Europe and the United States (9).

Despite increasing evidence of the high efficacy of radioimmunotherapy in NHL, the low toxicities, and approval by the Food and Drug Administration and the European Medicines Agency, these therapies are seldom used. A front-page article in the New York Times from July 14, 
2007, discussed innovative new treatments, market factors, and health politics in the choices of treatment for NHL. This article claimed that in the United States the options for drugs to treat cancer, such as radioimmunotherapy, are limited in their use because of market forces (10).

A recent editorial in the Journal of Clinical Oncology highlights the remarkably successful results of radioimmunotherapy in NHL. However, the authors also identified the gross underuse of this highly effective therapy for patients with NHL (11).

We recently published a survey of medical oncologists and hematologists in the United States as related to radioimmunotherapy use in lymphoma (12). Despite the low use of radioimmunotherapy by medical oncologists and hematologists, we generally found a positive perception of radioimmunotherapy in this group of physicians. It seemed that logistic and economic hurdles were their major concerns. We concluded that it appears crucial to streamline the referral process, enhance collaboration between specialists, and develop economic incentives (or reduce financial disincentives) for the referring physicians.

Several comments by the referring physicians discussed the complicated referral process and low interest of nuclear physicians in treating NHL with radioimmunotherapy. Representative of the remarks by other responders, a medical oncologist wrote: "The major hurdle is in the logistics since our nuclear medicine physicians are not very interested in therapy."

In this report we now present a nationwide study of health professionals working in nuclear medicine or radiation oncology. We surveyed these health professionals about their opinions of radioimmunotherapy in NHL. We emphasized their perceptions regarding the treatment in broad areas of evidence-based efficacy and logistical barriers and their economic concerns. We also compared their opinions with the perceptions of the medical oncologists and hematologists in the United States. In conducting these surveys, our aim was to identify barriers to the use of radioimmunotherapy in NHL and to further increase the potential availability of these promising drugs for our patients with cancer.

\section{MATERIALS AND METHODS}

We created a survey to assess various aspects of the treatment process using radioimmunotherapy (Fig. 1) in patients with NHL. We assessed our survey in a pilot setting in our own institution at the Johns Hopkins Hospital. The questions focused on treatment numbers, referral numbers, perceived indications, treatment settings, and possible concerns of the nuclear physician or radiation oncologist administering the treatment. The survey was distributed using the databases of the Society of Nuclear Medicine (SNM) members and other databases of radiation oncologists. We used an e-mail-based electronic survey tool (SurveyGizmo; Widgix, LLC).

A questionnaire with 13 broad questions was used in this survey. The question formats included multiple-choice, multipleselection, a table of multiple-choice options, and an opportunity for free text. The strength of response was typically assessed by a 5-point scale (1-5) for a given question. The complete survey is provided in the supplemental material (available online only at http://jnm.snmjournals.org). The Institutional Review Board of the Johns Hopkins University approved the study protocol. Potential responders were informed of the principal investigator's name but were not told about any possible financial interest of the principal investigator in either of these forms of therapy.

We calculated means and SD. For the multivariate analyses, logistic regression or proportional odds models were fitted for binary or ordered categoric response variables, and linear regression models were fitted for physician concern variables. These models will adjust for potential confounding covariates such as geographics and practice type.

\section{RESULTS}

\section{Demographic Characteristics of Study Participants}

Invitations $(13,221)$ to participate in our survey were sent to SNM members and radiation oncologists identified from various sources. A total of 2,128 persons opened the survey; 1,507 individuals abandoned the survey, and 613 individuals responded to the survey. A map of the geographic location of the responders is provided in Figure 1. The calculated completion rate was $29.1 \%$. We resent the survey twice if there was no response after each 7-d period and stopped after $31 \mathrm{~d}$ overall. There were 542 SNM members and 71 additional radiation oncologists who responded to the survey. From 542 SNM members, 304 were nuclear physicians, 198 were nuclear technologists, and 40 were researchers affiliated with nuclear medicine research. Two hundred forty-five individuals worked in academic hospitals, 237 in nonacademic hospitals, 27 in multispecialty offices, 79 in private offices, and 25 in industry (Fig. 2).

\section{Treatment Numbers and Type of Referrals}

For the treatment of patients with lymphoma with CD20directed radioimmunotherapy, 262 responders to the survey reported performing no treatment with ${ }^{131} \mathrm{I}$-tositumomab or ${ }^{90}$ Y-ibritumomab tiuxetan in the last 24 mo, 170 reported 1-3 treatments, 88 reported 4-7 treatments, 52 reported 814 treatments, and 41 reported more than 15 treatments (Fig. 3). Three hundred fifty-one responders treated NHL with radioimmunotherapy. Two hundred eight responders $(33.93 \%)$ reported referrals from hematologists and oncologists from university hospitals, $143(23.32 \%)$ from nonacademic hospitals, 86 (14.03\%) from multispecialty offices, $163(26.59 \%)$ from private offices, and $22(3.6 \%)$ from patient self-referrals; $189(30.83 \%)$ responders had not treated any patients in the last 24 mo.

\section{Perceived Indications for Radioimmunotherapy}

One hundred forty-two (23\%) responders of the surveyed population perceived radioimmunotherapy with ${ }^{131} \mathrm{I}$ tositumomab or ${ }^{90} \mathrm{Y}$-ibritumomab tiuxetan to be a first-line indication, $350(42.9 \%)$ as second-line, $218(35.6 \%)$ as thirdline, $116(18.9 \%)$ later than third-line, and $144(23.5 \%)$ as consolidation after first-line therapy for patients with follicular lymphoma. Seventeen (2.8\%) responders thought this form of treatment should be used only for investigational 


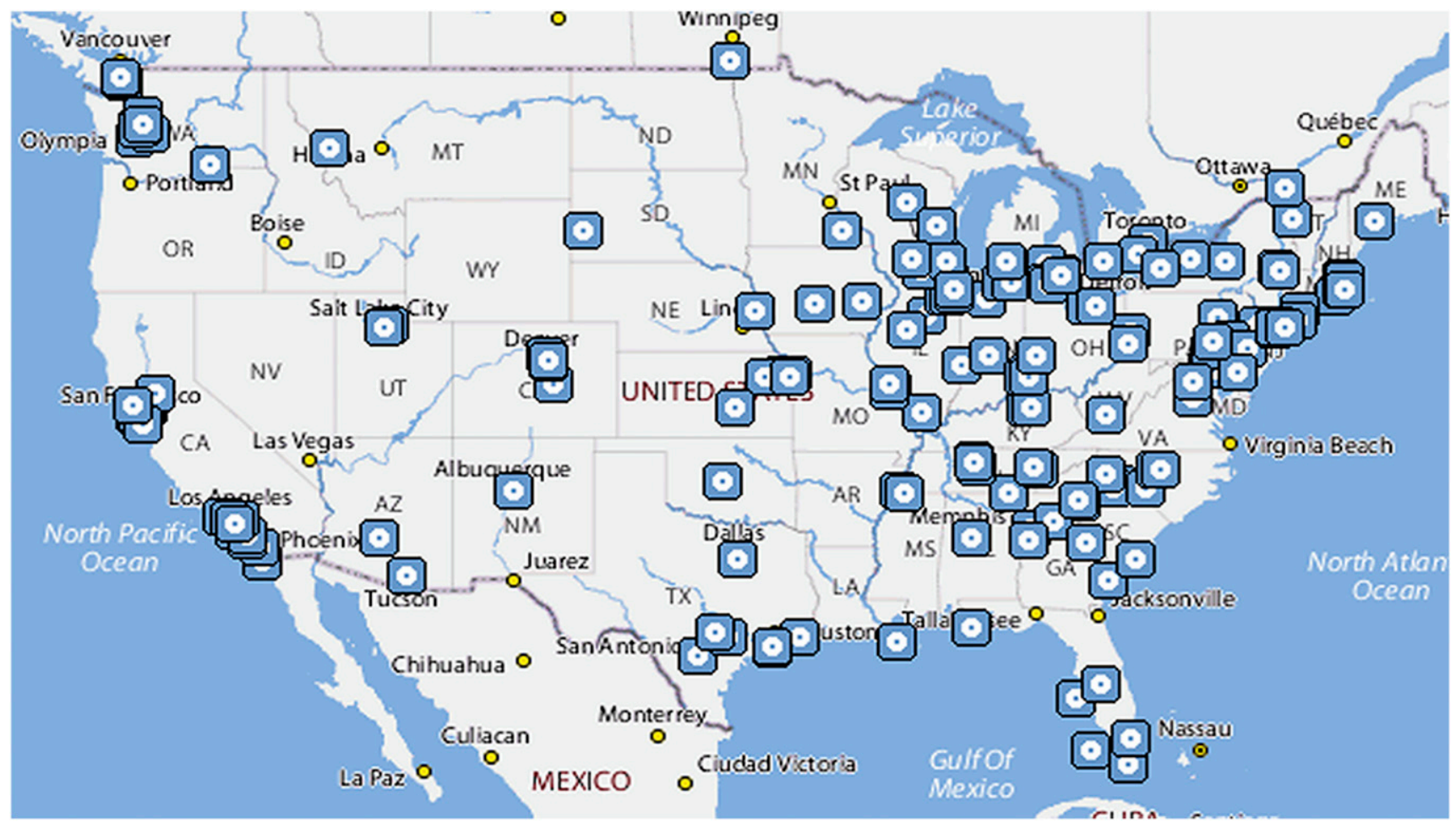

FIGURE 1. Geographic locations of responders to survey (Yahoo! MapMaker for Excel, version 1.02; Microsoft).

purposes, and 14 (2.3\%) did not see any clinical indication for ${ }^{131} \mathrm{I}$-tositumomab or ${ }^{90} \mathrm{Y}$-ibritumomab tiuxetan. Figure 4 summarizes the perceived indications for radioimmunotherapy.

\section{Type of Radiopharmaceutical Used, Treatment Setting, and Further Information}

One hundred eighty-three $(29.5 \%)$ of the responders to our survey used ${ }^{90}$ Y-ibritumomab tiuxetan exclusively, and $47(7.6 \%)$ of the responders used only ${ }^{131}$ I-tositumomab. One hundred fifty-three (24.9\%) responders used both radiopharmaceuticals. In the responders who treated NHL with radioimmunotherapy $(n=351), 163(46.44 \%)$ used only ${ }^{90}$ Y-ibritumomab tiuxetan, $44(12.54 \%)$ used only ${ }^{131} \mathrm{I}-$ tositumomab, and 144 (41.03\%) used both therapies. Three hundred forty $(77.1 \%)$ responders used ${ }^{90}$ Y-ibritumomab tiuxetan or ${ }^{131}$ I-tositumomab in an inpatient setting. Twenty-six (5.9\%) of the responders preferred an outpatient setting. Seventy-five $(12.1 \%)$ responders used ${ }^{131}$ I-tositumomab or ${ }^{90}$ Y-ibritumomab tiuxetan in an in- or outpatient setting. One hundred thirty-one (24.2\%) responders from the SNM group reported that their radiation oncologists were using unsealed sources in their institution. Two hundred eighty-one $(45.3 \%)$ responders reported that their institution accepted Medicare patients for ${ }^{90} \mathrm{Y}$-ibritumomab tiuxetan or ${ }^{131}$ I-tositumomab; $40.41 \%$ of responders and $35.2 \%$ of the involved physicians did not know if their institution accepted Medicare patients for radioimmunotherapy. Of the responders, $14.2 \%$ reported that their institution did not accept Medicare patients for radioimmunotherapy.
Four hundred sixty-two $(85.24 \%)$ responders used radioactive iodine for treatment of thyroid diseases.

\section{Concerns Raised Regarding Anti-CD20 Radioimmunotherapy}

The surveyed population rated their concerns about radioimmunotherapy for NHL from 1 (no concern) to 5 (major concern); 13 factors were rated. These factors, listed in decreasing order of concern, are summarized in Table 1.

\section{Emerging Patterns of Concerns After Subgroup Analyses}

Academia Versus Nonacademia. Responders from academic centers had treated more NHL patients with radio-

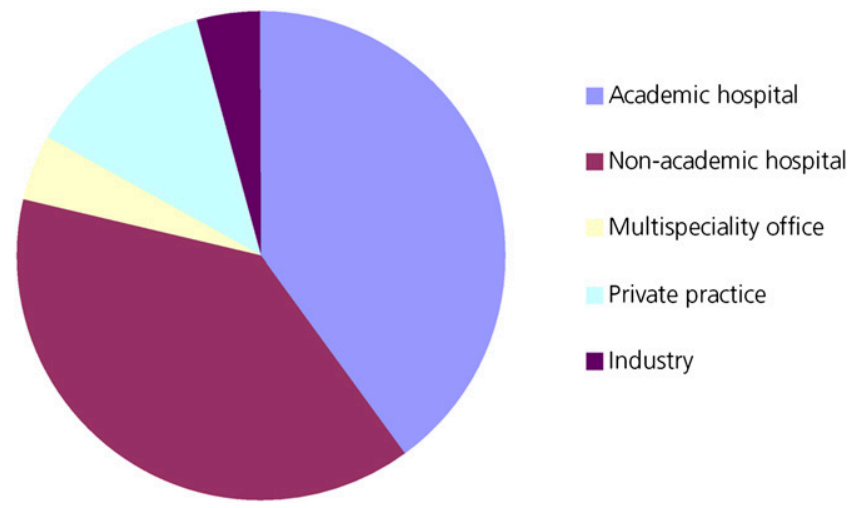

FIGURE 2. Two hundred forty-five (39.9\%) responders came from academic hospitals, 237 (38.7\%) from nonacademic hospitals, 27 (4.4\%) from multispecialty offices, $79(12.8 \%)$ from private practices, and $25(4.1 \%)$ from industry. 


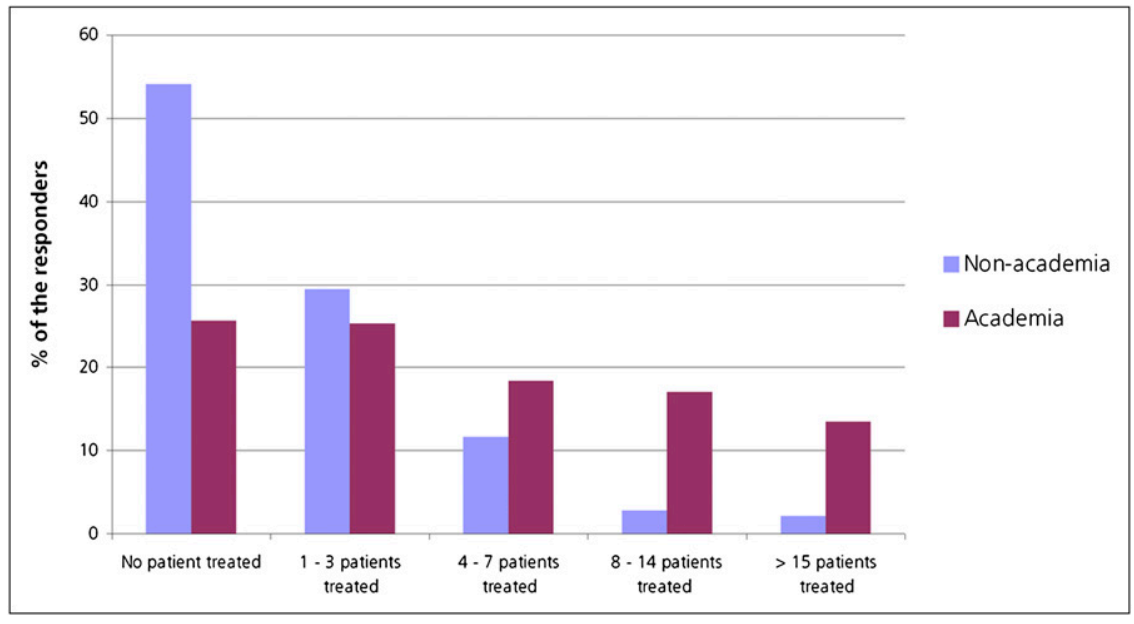

FIGURE 3. Percentage of responders who treated patients with $\mathrm{NHL}$ with radioimmunotherapy, analyzed by academia and nonacademia. For treatment of patients with lymphoma with CD20-directed radioimmunotherapy, 262 responders reported no treatment with ${ }^{131} \mathrm{I}$-tositumomab or ${ }^{90} \mathrm{Y}$ ibritumomab tiuxetan in last $24 \mathrm{mo}, 170$ reported 1-3 treatments, 88 reported 4-7 treatments, 52 reported 8-14 treatments, and 41 reported more than 15 treatments. immunotherapy $(P<0.01)$, thought radioimmunotherapy in lymphoma should be used in earlier lines (first and second lines) $(P<0.01)$, and had significantly fewer concerns about the poor payment of the radiolabeling $(P<0.01)$ and administration processes $(P<0.01)$. The responders from nonacademic centers had significantly more concerns about ${ }^{131}$ I-tositumomab or ${ }^{90} \mathrm{Y}$-ibritumomab tiuxetan taking too much time from their practice $(P<0.01)$. The radiation safety $(P<0.01)$ and dosimetry procedures were further concerns $(P<0.01)$.

Decision to Treat Patients with Lymphoma with Radioimmunotherapy in Last 24 Months. Compared with the subgroup of responders who did treat with radioimmunotherapy in the last $24 \mathrm{mo}$, the subgroup that did not was significantly concerned that radioimmunotherapy administration took too much time $(P<0.01)$, was concerned about radiation safety $(P<0.01)$ and the dosimetry procedure $(P<0.01)$, and thought that the administration of ${ }^{131} \mathrm{I}-$ tositumomab $(P=0.01)$ and ${ }^{90}$ Y-ibritumomab tiuxetan $(P=0.02)$ was too complicated. Nuclear physicians who did not treat with radioimmunotherapy also thought that they were not sufficiently trained to administer potentially myeloablative therapies $(P<0.01)$ (Table 2$)$.
Referral Indication. Responders who thought that ${ }^{131} \mathrm{I}$ tositumomab or ${ }^{90}$ Y-ibritumomab tiuxetan should not be used in first- or second-line treatment in patients with lymphoma also thought that the administration of ${ }^{131}$ I-tositumomab $(P=0.05)$ and ${ }^{90}$ Y-ibritumomab tiuxetan $(P=0.07)$ was too complicated. Responders who saw consolidation treatment as a major indication had significantly more concerns than oncologists and hematologists who preferred to treat by themselves with nonradioactive compounds $(P<0.01)$.

Predicted Future Growth of Radioimmunotherapy with ${ }^{131}$ I-Tositumomab or ${ }^{90}$ Y-Ibritumomab Tiuxetan in NHL. Responders who thought positively about radioimmunotherapy in NHL (that it would probably grow or would definitively grow in the future) had significantly more concerns about ${ }^{90}$ Y-ibritumomab tiuxetan or ${ }^{131}$ I-tositumomab being too expensive $(P<0.01)$ and the low number of randomized trials $(P<0.01)$ and tended to think that nuclear physicians were not trained for the administration of myeloablative therapies $(P=0.06)$ (Table 3$)$.

Treatment of Thyroid Diseases with Radioactive Iodine. Responders who treated patients with radioactive iodine treated significantly more patients with ${ }^{90}$ Y-ibritumomab tiuxetan or ${ }^{131}$ I-tositumomab $(P<0.01)$, were more con-

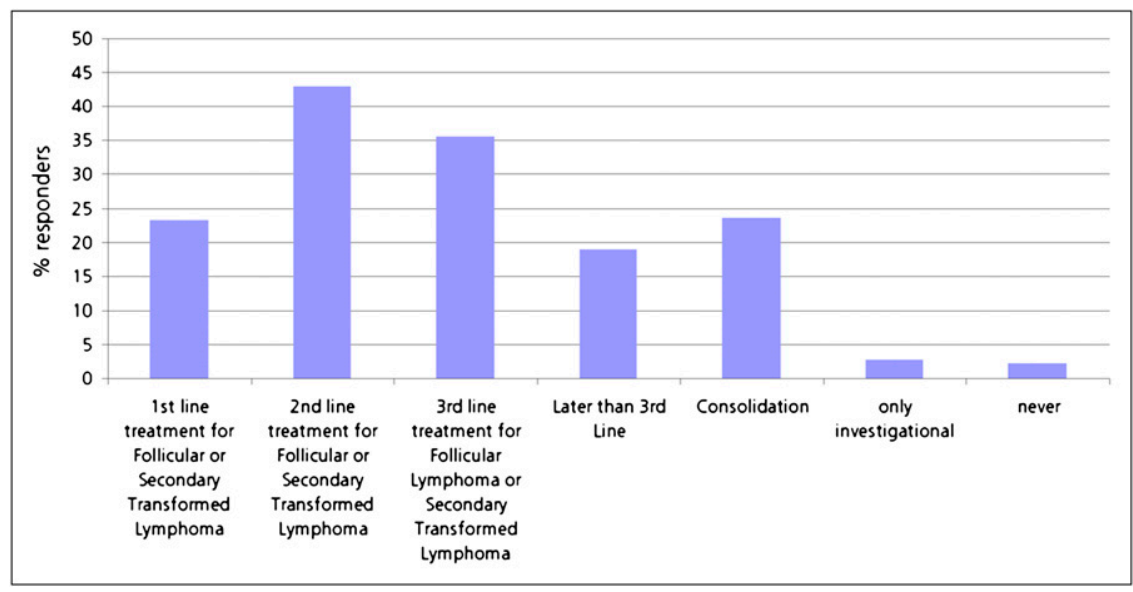

FIGURE 4. Percentage of perceived indications for radioimmunotherapy in $\mathrm{NHL}$ by responder. 
TABLE 1

Concerns About Radioimmunotherapy for NHL According to Surveyed Population

\begin{tabular}{|c|c|}
\hline Concern & $\begin{array}{l}\text { Mean rating } \pm \\
\quad \text { SD }\end{array}$ \\
\hline Oncologists and hematologists prefer to treat by themselves with nonradioactive compounds & $3.42 \pm 1.49$ \\
\hline${ }^{131}$ I-tositumomab or ${ }^{90} \mathrm{Y}$-ibritumomab tiuxetan treatment is too expensive & $3.41 \pm 1.34$ \\
\hline $\begin{array}{l}{ }^{90} \text {-ibritumomab tiuxetan or }{ }^{131} \text { I-tositumomab therapy provides poor payment for the time and effort in the } \\
\text { administration process }\end{array}$ & $2.91 \pm 1.53$ \\
\hline $\begin{array}{l}90 \text { Y-ibritumomab tiuxetan or }{ }^{131} \mathrm{I} \text {-tositumomab therapy provides poor payment for the time and effort in the } \\
\text { radiolabeling process }\end{array}$ & $2.51 \pm 1.41$ \\
\hline${ }^{131}$ I-tositumomab or ${ }^{90} Y$-ibritumomab tiuxetan treatment does not have enough reported randomized studies & $2.49 \pm 1.2$ \\
\hline $\begin{array}{l}\text { Most nuclear physicians are not trained to administer potential myeloablative therapies such as }{ }^{90} \text { Y-ibritumomab } \\
\text { tiuxetan or }{ }^{131} \text {-tositumomab }\end{array}$ & $2.48 \pm 1.35$ \\
\hline $\begin{array}{l}\text { Concerns exist that subsequent treatments will not be possible after }{ }^{131} \text { I-tositumomab or }{ }^{90} \text { Y-ibritumomab tiuxetan } \\
\text { administration }\end{array}$ & $2.47 \pm 1.14$ \\
\hline${ }^{131}$ I-tositumomab administration is too complicated & $2.46 \pm 1.38$ \\
\hline $\begin{array}{l}\text { There might be unexpected late side effects of }{ }^{131} \text {-tositumomab or }{ }^{90} Y \text {-ibritumomab tiuxetan treatment } \\
\text { (myelodysplastic syndrome) }\end{array}$ & $2.36 \pm 1.04$ \\
\hline${ }^{131}$ |-tositumomab or ${ }^{90} Y$-ibritumomab tiuxetan take too much time from my practice & $2.12 \pm 1.21$ \\
\hline We have concerns about the dosimetry procedure & $2.01 \pm 1.17$ \\
\hline We have concerns about radiation safety & $1.88 \pm 1.11$ \\
\hline${ }^{90} Y$-ibritumomab tiuxetan administration is too complicated & $1.78363 \pm 0.99$ \\
\hline
\end{tabular}

cerned about the high cost of ${ }^{90}$ Y-ibritumomab tiuxetan or ${ }^{131}$ I-tositumomab $(P<0.01)$, and thought more positively about the future growth of radioimmunotherapy in patients with lymphoma $(P=0.09)$.
Treatment with Unsealed Sources by Radiation Oncologists at Same Institution. Nuclear medicine responders who worked with radiation oncologists using unsealed sources at the same institution tended to have more

TABLE 2

Concerns About Treatment of NHL Patients with ${ }^{90}$ Y-Ibritumomab Tiuxetan or ${ }^{131}$ I-Tositumomab Grouped by Treatment or No Treatment

\begin{tabular}{|c|c|c|c|}
\hline \multirow[b]{2}{*}{ Concern } & \multicolumn{2}{|c|}{ Mean rating } & \multirow[b]{2}{*}{$P$} \\
\hline & $\begin{array}{l}\text { Does } \\
\text { treat }\end{array}$ & $\begin{array}{l}\text { Does not } \\
\text { treat }\end{array}$ & \\
\hline We have concerns about the radiation safety & 1.86 & 1.87 & 0.77 \\
\hline${ }^{90}$-ibritumomab tiuxetan administration is too complicated in general & 1.88 & 1.72 & 0.83 \\
\hline We have concerns about the dosimetry procedure & 2.01 & 2.00 & 0.45 \\
\hline $\begin{array}{l}\text { There might be unexpected late side effects of }{ }^{131} \text {-tositumomab or }{ }^{90} \mathrm{Y} \text {-ibritumomab tiuxetan treatment } \\
\text { (myelodysplastic syndrome) }\end{array}$ & 2.28 & 2.38 & 0.47 \\
\hline${ }^{131}$-tositumomab or ${ }^{90} \mathrm{Y}$-ibritumomab tiuxetan takes too much time from my practice in general & 2.29 & 2.01 & 0.22 \\
\hline $\begin{array}{l}\text { Most nuclear physicians are not trained to administer potential myeloablative therapies such as }{ }^{90} \mathrm{Y}- \\
\text { ibritumomab tiuxetan or }{ }^{131} \text { I-tositumomab* }\end{array}$ & 2.39 & 2.51 & 0.06 \\
\hline $\begin{array}{l}90 Y \text {-ibritumomab tiuxetan or }{ }^{131} \mathrm{I} \text {-tositumomab therapy provides poor payment for the time and effort in the } \\
\text { radiolabeling process }\end{array}$ & 2.49 & 2.51 & 0.33 \\
\hline $\begin{array}{l}\text { Concerns exist that subsequent treatments will not be possible after }{ }^{131} \text { I-tositumomab or }{ }^{90} \text { Y-ibritumomab } \\
\text { tiuxetan administration }\end{array}$ & 2.53 & 2.46 & 0.22 \\
\hline $\begin{array}{l}{ }^{131} \text { I-tositumomab or }{ }^{90} \text { Y-ibritumomab tiuxetan treatment does not have enough reported randomized } \\
\text { studies }{ }^{\star}\end{array}$ & 2.58 & 2.46 & $<0.01$ \\
\hline${ }^{131}$ I-tositumomab administration is too complicated in general & 2.62 & 2.41 & 0.27 \\
\hline $\begin{array}{l}90 Y \text {-ibritumomab tiuxetan or }{ }^{131} \mathrm{I} \text {-tositumomab therapy provides poor payment for the time and effort in the } \\
\text { administration process }\end{array}$ & 3.01 & 2.88 & 0.62 \\
\hline Oncologists and hematologists prefer to treat by themselves with nonradioactive compounds & 3.47 & 3.43 & 0.34 \\
\hline${ }^{131}$ I-tositumomab or ${ }^{90} \mathrm{Y}$-ibritumomab tiuxetan treatment is too expensive ${ }^{*}$ & 3.60 & 3.34 & $<0.01$ \\
\hline $\begin{array}{l}{ }^{*} \text { Considered significant difference. } \\
P<0.05 \text { was considered significant. }\end{array}$ & & & \\
\hline
\end{tabular}




\begin{tabular}{|c|c|c|c|}
\hline \multirow[b]{2}{*}{ Concern } & \multicolumn{2}{|c|}{ Mean rating } & \multirow[b]{2}{*}{$P$} \\
\hline & $\begin{array}{c}\text { Positive } \\
\text { future }(\geq 4)\end{array}$ & $\begin{array}{l}\text { Negative } \\
\text { future }(<4)\end{array}$ & \\
\hline${ }^{90} \mathrm{Y}$-Ibritumomab tiuxetan administration is too complicated in general ${ }^{*}$ & 1.62 & 2.06 & 0.02 \\
\hline We have concerns about the radiation safety* & 1.69 & 2.19 & $<0.01$ \\
\hline We have concerns about the dosimetry procedure* & 1.82 & 2.33 & $<0.01$ \\
\hline${ }^{131}$ I-tositumomab or ${ }^{90} \mathrm{Y}$-ibritumomab tiuxetan take too much time from my practice in general ${ }^{\star}$ & 1.94 & 2.39 & $<0.01$ \\
\hline $\begin{array}{l}\text { Most nuclear physicians are not trained to administer potentially myeloablative therapies such as } \\
{ }_{90} \text {-ibritumomab tiuxetan or }{ }^{131} \text { I-tositumomab* }\end{array}$ & 2.27 & 2.87 & $<0.01$ \\
\hline $\begin{array}{l}\text { There might be unexpected late side effects of }{ }^{131} \text {-tositumomab or }{ }^{90} \text { Y-ibritumomab tiuxetan } \\
\text { treatment (myelodysplastic syndrome) }\end{array}$ & 2.30 & 2.44 & 0.45 \\
\hline $\begin{array}{l}\text { Concerns exist that subsequent treatments will not be possible after }{ }^{131} \text {-tositumomab or }{ }^{90} \mathrm{Y} \text { - } \\
\text { ibritumomab tiuxetan administration }\end{array}$ & 2.41 & 2.60 & 0.33 \\
\hline $\begin{array}{l}90 \text { Y-ibritumomab tiuxetan or }{ }^{131} \text { I-tositumomab therapy provides poor payment for the time and } \\
\text { effort in the radiolabeling process }\end{array}$ & 2.42 & 2.64 & 0.17 \\
\hline $\begin{array}{l}{ }^{131} \mathrm{I} \text {-tositumomab or }{ }^{90} \mathrm{Y} \text {-ibritumomab tiuxetan treatment does not have enough reported } \\
\text { randomized studies }\end{array}$ & 2.49 & 2.52 & 0.74 \\
\hline${ }^{131}$ I-tositumomab administration is too complicated in general ${ }^{\star}$ & 2.56 & 2.35 & 0.01 \\
\hline $\begin{array}{l}90 \text { Y-ibritumomab tiuxetan or }{ }^{131} \mathrm{I} \text {-tositumomab therapy provides poor payment for the time and } \\
\text { effort in the administration process }\end{array}$ & 2.94 & 2.90 & 0.3 \\
\hline Oncologists and hematologists prefer to treat by themselves with nonradioactive compounds & 3.48 & 3.38 & 0.2 \\
\hline${ }^{131}$ I-tositumomab or ${ }^{90} \mathrm{Y}$-ibritumomab tiuxetan treatment is too expensive & 3.51 & 3.27 & 0.18 \\
\hline $\begin{array}{l}{ }^{*} \text { Considered significant difference. } \\
P<0.05 \text { was considered significant. }\end{array}$ & & & \\
\hline
\end{tabular}

concerns that nuclear physicians may not be properly trained to administer potentially myeloablative therapies $(P=0.08)$.

Inpatient Treatment of Patients with Radioimmunotherapy. Responders who treated NHL patients on an inpatient basis tended to have significantly more concerns about dosimetry procedures $(P=0.01)$ and radiation safety $(P=0.08)$.

Using Either ${ }^{131}$ I-Tositumomab or ${ }^{90} Y$-Ibritumomab Tiuxetan. Responders from academic centers were more likely to use ${ }^{131}$ I-tositumomab $(P<0.01)$; responders from nonacademic centers used more ${ }^{90}$ Y-ibritumomab tiuxetan $(P<$ 0.01). Responders using only ${ }^{90}$ Y-ibritumomab tiuxetan perceived ${ }^{131}$ I-tositumomab as too complicated $(P<0.01)$, and responders using only ${ }^{131} \mathrm{I}$-tositumomab were concerned that ${ }^{90}$ Y-ibritumomab tiuxetan administration was too complicated $(P=0.01)$.

Radiation Oncologists Versus Nuclear Physicians. Radiation oncologists tended to be more concerned than nuclear physicians that ${ }^{131}$ I-tositumomab or ${ }^{90}$ Y-ibritumomab tiuxetan administration took too much time from their practice $(P=0.094)$ and that subsequent treatments would not be possible after the administration of either radiopharmaceutical $(P=0.024)$.

Radiation Oncologists and Nuclear Physicians Versus Medical Oncologists and Hematologists. We compared the concerns from the current survey with our past survey of the medical oncologists and hematologists throughout the United States (10). The medical oncologists were signifi- cantly less concerned about the high price of ${ }^{90} \mathrm{Y}$-ibritumomab tiuxetan and ${ }^{131}$ I-tositumomab $(P<0.01)$, but we were more concerned about the late side effects (myelodysplastic syndrome) of ${ }^{90} \mathrm{Y}$-ibritumomab tiuxetan or ${ }^{131}$ I-tositumomab $(P<0.01)$.

\section{Predicted Future Growth for Radioimmunotherapy with ${ }^{131}$ I-Tositumomab or ${ }^{90}$ Y-Ibritumomab Tiuxetan Radioimmunotherapy for NHL}

The responders had an opportunity to answer a question regarding their prediction of the potential future growth of radioimmunotherapy for NHL, rating the potential from 1 (it will not grow) to 5 (it will grow). One $(0.17 \%)$ responder to the survey thought radioimmunotherapy for NHL would definitely not grow in the future, $37(6.33 \%)$ thought it would probably not grow in the future, 151 $(25.85 \%)$ were uncertain about the growth of radioimmunotherapy for NHL in the future, $173(29.6 \%)$ thought it would probably grow, and $222(38.01 \%)$ thought radioimmunotherapy in NHL with ${ }^{131}$ I-tositumomab and ${ }^{90} \mathrm{Y}-$ ibritumomab tiuxetan would definitely grow in importance in the future. Again, comparing the current survey with our past survey of medical oncologists and hematologists, there was a significantly brighter outlook $(P<0.0001)$ among the nuclear physicians (mean, 4.0; SE, 0.04) and radiation oncologists (mean, 3.93; SE, 0.12) than among the medical oncologists and hematologists (mean, 3.64; SE, 0.08) (Fig. 5). 
FIGURE 5. Predicted future growth of radioimmunotherapy in $\mathrm{NHL}$. Percentage of responders is from current survey and our last survey of medical oncologists and hematologists (12). HemOnc $=$ hematologists and oncologists; MedOnc = medical oncologists; $\mathrm{NM}=$ nuclear medicine physicians; RadOnc $=$ radiation oncologists.

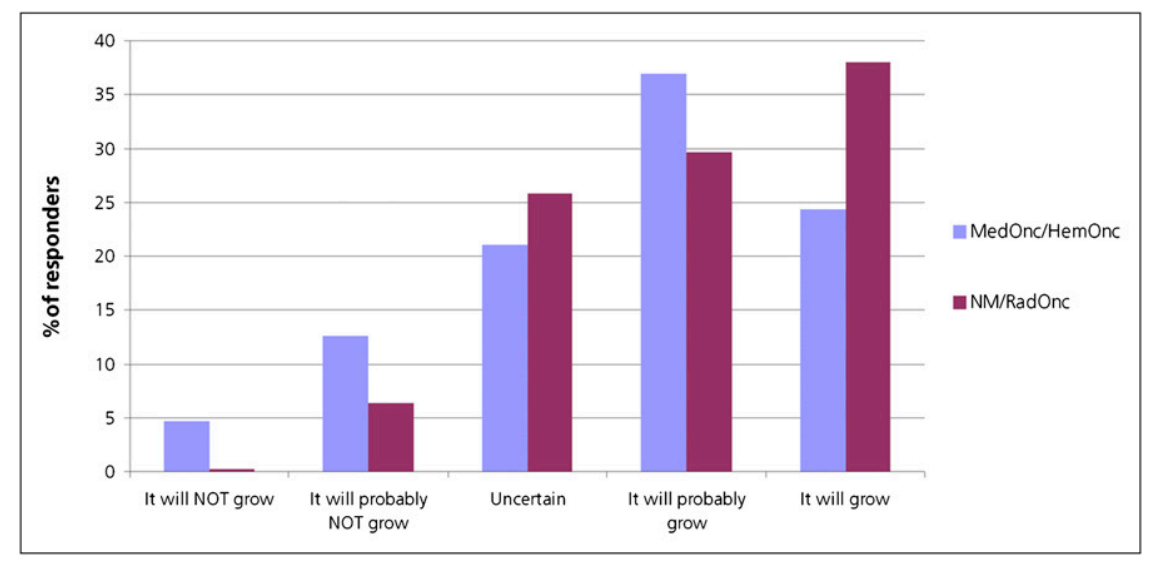

\section{Do You Have Any Further Comments Pertaining to Treatment of NHL with ${ }^{131}$ I-Tositumomab or ${ }^{90}$ Y-Ibritumomab Tiuxetan?}

This essay text field was open for further comments. One hundred forty-six $(23.6 \%)$ of 613 responders added one or more specific comments. Comments covered primarily medical $(n=5)$, scientific $(n=18)$, logistic $(n=25)$, economic $(n=56)$, and educational $(n=16)$ issues, with most dealing with economic concerns. These comments addressed mainly the competing economic interests of the medical oncologists and hematologists versus the physicians in the administration of radioimmunotherapy.

Major economic concerns were also raised about the poor reimbursement and loss of money by the administering facility. Two representative economic comments were, "Expense is the only real problem. I lose $\$ 3,000$ on average if I treat an outpatient, so now I refuse to do it" and "After spending over $1 \mathrm{y}$ to get the radioimmunotherapy program off the ground it was shut down due to the huge monetary loss in treating NHL patients." Twenty-five responders commented on logistic problems. One responder wrote, "There are too many radiation safety concerns; our nurses refuse to care for radioimmunotherapy patients in our hospital." Another responder wrote, "Our radiology department did not wish to get involved with Bexxar or Zevalin, so by default the Radiation Oncologists use Zevalin at our institution."

Eighteen responders were concerned about the lack of evidence from phase III trials and head-to-head comparisons of radioimmunotherapy versus nonlabeled antibodies; 16 responders pointed out the poor educational effort and lack of knowledge of patients and referring physicians. Various responders wrote, "Patients need to be educated to request this therapy from their oncologists earlier in the course of disease, as private oncologists do not have a financial incentive to refer for this therapy" and "One major problem has been that nuclear medicine physicians have not provided support and dedication in providing the resources needed for RIT.” Only 5 responders outlined medical concerns. Their main concern was the prolonged myelosuppression.

\section{DISCUSSION}

Radioimmunotherapy with ${ }^{131} \mathrm{I}$-tositumomab and ${ }^{90} \mathrm{Y}-$ ibritumomab tiuxetan is approved by the Food and Drug Administration for follicular and secondary transformed lymphoma after first-line therapy. In September 2009, Zevalin was approved by the Food and Drug Administration as part of first-line consolidation therapy after primary immune- or chemotherapy in both the United States and Europe. Current guidelines of the National Comprehensive Cancer Network (version 1.2010 (13)) list radioimmunotherapy as a first-line therapy, as a first-line therapy for the elderly or infirm, as a first-line consolidation agent, and as a second-line and subsequent therapy agent. However, despite the increasing evidence and acceptance by the oncology community of the efficacy and safety of radioimmunotherapy in NHL, these forms of therapy are not routinely used. This study is the first, to our knowledge, to analyze the perceptions of professionals in nuclear medicine and radiation oncology in the United States.

A completion rate, which represents the ratio of opened and completed surveys, of $29.2 \%$ is within the reference range for an e-mail-based survey (14). Of the responders, $262(42.2 \%)$ had not treated any lymphoma patients with radioimmunotherapy. We focused mainly on the concerns that prevented them from treating any NHL patients with radioimmunotherapy. The responders who did not treat any patients with lymphoma in the last 24 mo had significantly more logistic and educational concerns. Their main concerns were radioimmunotherapy taking too much time from their practice $(P<0.01)$, radiation safety $(P<0.01)$, the dosimetry procedure $(P<0.01)$, and the complicated administration process of both radiopharmaceuticals $(P<0.01)$.

We also analyzed the subgroup of nuclear physicians who did not treat any patients. The subgroup analysis showed that nuclear physicians who did not treat with radioimmunotherapy felt that they were not trained to administer potentially myeloablative therapies $(P<0.01)$. These observations show that we not only have to streamline the radioimmunotherapy administration process but also have to provide sufficient training for the treating physicians. 
Academic centers do treat significantly more patients. It seems that these large centers have the ability to absorb these complicated and potentially resource-consuming processes and that they are able to provide the needed training and expertise for radioimmunotherapy administration. One responder wrote, "Training in Zevalin and Bexxar is practically nonexistent," and a different responder wrote, "Radioimmunotherapy is just too complicated for a small-volume practice." Therefore, we need specific processes to facilitate radioimmunotherapy administration and specific training courses and facilities. It must be a goal that future nuclear physicians not only feel that they are a part of the diagnostic workup of patients with lymphoma but also are as comfortable and confident as the treating physicians in these diseases. A strong indicator of the lack of self-confidence of nuclear physicians as treating specialists is the fact that nuclear physicians who work with radiation oncologists using radionuclides for therapy think that they are not sufficiently trained to administer potential myeloablative therapies $(P<0.01)$. In our opinion, this is one of the most important factors affecting why radioimmunotherapy in NHL is underused.

Two of the top 5 concerns were the poor reimbursement for administration and reimbursement for radiolabeling. The subgroup analyses revealed that these were strong predictors of whether to treat. This finding is congruent with the recent observation that medical oncologists and hematologists who want to refer patients for radioimmunotherapy often do not find an appropriate partner in nuclear medicine. In our recent survey, 1 responder from medical oncology wrote, "I refer patients but the faculty always recommends chemotherapy, even when it seems like radioimmunotherapy would be a better choice." The great importance of reimbursement in the decision not to treat NHL patients with radioimmunotherapy, and the lack of treatment sites, show the urgent need to generate a more adequate financial incentive for treatment. This incentive will most likely be accomplished by adjusting the reimbursement for the physicians administering radioimmunotherapy in proportion to the work involved. Overall, we see an economic disincentive for the referring and treating physicians-another important finding explaining why radioimmunotherapy in NHL is underused.

The biggest concern overall is that oncologists and hematologists prefer to treat by themselves with nonradioactive compounds. This concern was even higher $(P<0.01)$ in the subgroup analysis of physicians who saw an indication for radioimmunotherapy in consolidation. With the recent publication of the interim data of the Primary Rituximab and Maintenance study at the American Society of Clinical Oncology meeting in 2010, a significant improvement in the primary endpoint, PFS, for rituximab (Rituxan; Biogen Idec/Genentech) maintenance was shown in patients with NHL after rituximab therapy containing first-line chemo- or immunotherapy (15). However, the randomized FIT consolidation trial with ${ }^{90}$ Y-ibritumomab tiuxetan after first-line therapy showed impressive differences in the PFS of NHL patients, with a single dose of ${ }^{90}$ Y-ibritumomab tiuxetan prolonging progression of the tumor by $2 \mathrm{y}$. The current competition of labeled and unlabeled antibody treatments in the consolidation of NHL further underlines the need to streamline all processes that are connected to radioimmunotherapy in NHL patients.

The second highest concern in the current survey was the high expense of ${ }^{90} \mathrm{Y}$-ibritumomab tiuxetan and ${ }^{131} \mathrm{I}$ tositumomab. This concern was even significantly higher in the group with a positive outlook for the future of radioimmunotherapy in NHL. The cost for combined immuno- and chemotherapy with rituximab, cyclophosphamide, doxorubicin, vincristine, and prednisone is about $\$ 17,000$ (16). This overall cost is comparable with the reimbursement for radioimmunotherapy by Medicare (17). However, because of the nature of radioimmunotherapy as a 1-shot treatment, the treating physician could have a potential financial risk to absorb if the therapy is not given. Here, we need secure safeguards imposed by safety committees and industry, with possibly better financial assurances and protections for the treating physicians.

\section{CONCLUSION}

We see a generally positive perception for radioimmunotherapy in the responders from the surveyed population. However, economic and logistic considerations seem extremely important. Most responders, especially from nonacademic centers, have no economic incentive to treat with radioimmunotherapy in NHL. They are even more fearful of potential monetary loss by treating NHL patients with ${ }^{90}$ Y-ibritumomab tiuxetan or ${ }^{131}$ I-tositumomab. Nearly 55\% of the responders incorrectly believed that anti-CD20 radioimmunotherapy agents were not approved for payment by U.S. Medicare. These major concerns demonstrate the importance of adjusting the reimbursement strategies in the administration process of radioimmunotherapy. It also seems important to develop safeguards to protect physicians from financial risks associated with purchase of the therapeutic drug coupled with failure to be able to deliver the treatment.

Lack of training in the use of radioimmunotherapy is another important factor why some of our responders refused to or did not treat NHL patients with radioimmunotherapy. Here, we need new concepts about how to integrate this training into current nuclear medicine educational concepts and need clearly to invest in reeducating the physician workforce. A solution might be a subspecialty of nuclear medicine with emphasis on emerging therapies, including radioimmunotherapy, with training requirements and examination.

It seems crucial for the future of radioimmunotherapy in NHL to broaden the scientific base of radioimmunotherapy in NHL and streamline the overall logistic, economic, and training processes. Clearly, a well-educated and engaged base of physician practitioners is essential. There are several 
new interesting radioimmunotherapy and other radioactive compounds under clinical development. For successful integration of these compounds into the clinical arena, we need to focus not only on scientific evidence but also on logistic and economic issues.

\section{DISCLOSURE STATEMENT}

The costs of publication of this article were defrayed in part by the payment of page charges. Therefore, and solely to indicate this fact, this article is hereby marked "advertisement" in accordance with 18 USC section 1734.

\section{ACKNOWLEDGMENTS}

Dr. Richard L. Wahl is one of the inventors of anti-CD20 radioimmunotherapy and receives payments from the University of Michigan as part of a patent licensing agreement related to royalties from the U.S. sales of the radioimmunotherapy agents ${ }^{131}$ I-tositumomab and ${ }^{90}$ Y-ibritumomab tiuxetan. This arrangement has been disclosed to and is managed by the JHU COI Committee.

\section{REFERENCES}

1. Cheson BD, Leonard JP. Monoclonal antibody therapy for B-cell non-Hodgkin's lymphoma. N Engl J Med. 2008;359:613-626.

2. Kaminski MS, Zasadny KR, Francis IR, et al. Radioimmunotherapy of B-cell lymphoma with [ $\left.{ }^{131} \mathrm{I}\right]$ anti-B1 (anti-CD20) antibody. N Engl J Med. 1993;329: 459-465.

3. Vose JM, Wahl RL, Saleh M, et al. Multicenter phase II study of iodine-131 tositumomab for chemotherapy-relapsed/refractory low-grade and transformed low-grade B-cell non-Hodgkin's lymphomas. J Clin Oncol. 2000;18:1316-1323.

4. Kaminski MS, Tuck M, Estes J, et al. ${ }^{131} \mathrm{I}$-tositumomab therapy as initial treatment for follicular lymphoma. N Engl J Med. 2005;352:441-449.

5. Witzig TE, Gordon LI, Cabanillas F, et al. Randomized controlled trial of yttrium-90-labeled ibritumomab tiuxetan radioimmunotherapy versus rituximab immunotherapy for patients with relapsed or refractory low-grade, follicular, or transformed B-cell non-Hodgkin's lymphoma. J Clin Oncol. 2002;20:24532463.

6. Wiseman GA, Gordon LI, Multani PS, et al. Ibritumomab tiuxetan radioimmunotherapy for patients with relapsed or refractory non-Hodgkin lymphoma and mild thrombocytopenia: a phase II multicenter trial. Blood. 2002;99:43364342.

7. Witzig TE, Flinn IW, Gordon LI, et al. Treatment with ibritumomab tiuxetan radioimmunotherapy in patients with rituximab-refractory follicular non-Hodgkin's lymphoma. J Clin Oncol. 2002;20:3262-3269.

8. Link BK, Martin P, Kaminski MS, Goldsmith SJ, Coleman M, Leonard JP. Cyclophosphamide, vincristine, and prednisone followed by tositumomab and iodine-131-tositumomab in patients with untreated low-grade follicular lymphoma: eight-year follow-up of a multicenter phase II study. J Clin Oncol. 2010; 28:3035-3041.

9. Morschhauser F, Radford J, Van Hoof A, et al. Phase III trial of consolidation therapy with yttrium-90-ibritumomab tiuxetan compared with no additional therapy after first remission in advanced follicular lymphoma. J Clin Oncol. 2008;26: 5156-5164.

10. Berenson A. Market forces cited in lymphoma drugs' disuse. The New York Times. July 14, 2007. Available at: http://www.nytimes.com/2007/07/14/health/ 14lymphoma.html. Accessed March 11, 2011.

11. Illidge TM. Radioimmunotherapy of lymphoma: a treatment approach ahead of its time or past its sell-by date? J Clin Oncol. 2010;28:2944-2946.

12. Schaefer NG, Ma J, Huang P, Buchanan J, Wahl RL. Radioimmunotherapy in non-Hodgkin lymphoma: opinions of U.S. medical oncologists and hematologists. J Nucl Med. 2010;51:987-994.

13. National Comprehensive Cancer Network (NCCN). Non-Hodgkin's Lymphomas. NCCN Clinical Practice Guidelines in Oncology, v.1.2010. Fort Washington, PA: NCCN; 2010.

14. Hewson C, Yule P, Laurent D, Vogel C. Internet Research Methods: A Practical Guide for the Social and Behavioural Sciences. London, U.K.: Sage Publications Ltd.; 2003.

15. Salles GA, Seymour JF, Feugier P, et al. Rituximab maintenance for 2 years in patients with untreated high tumor burden follicular lymphoma after response to immunochemotherapy [abstract]. J Clin Oncol. 2010;28(suppl):15s.

16. Hornberger JC, Best JH. Cost utility in the United States of rituximab plus cyclophosphamide, doxorubicin, vincristine, and prednisone for the treatment of elderly patients with diffuse large B-cell lymphoma. Cancer. 2005;103: 1644-1651.

17. Centers for Medicare and Medicaid Services, Department of Health and Human Services. CMS Programs \& Information: Medicare. Available at: http://www. cms.hhs.gov/. Accessed March 11, 2011.

\section{Erratum}

In the article "Searching for Alternatives to Full Kinetic Analysis in ${ }^{18}$ F-FDG PET: An Extension of the Simplified Kinetic Analysis Method," by Hapdey et al. ( Nucl Med. 2011;52:634-641), the name of the third author was misspelled. The correct spelling is Joann M. Carson. The authors regret the error. 